\title{
The Operational Land Imager (OLI) and the Thermal Infrared Sensor (TIRS) on the Landsat Data Continuity Mission (LDCM)
}

Dennis Reuter, James Irons, Allen Lunsford, Matthew Montanero, Fernando

Pellerano, Cathleen Richardson, Ramsey Smith, Zelalem Tesfaye, Kurtis Thome

\section{Summary Text}

The Thermal Infrared Sensor (TIRS) instrument, provided by NASA's Goddard Space Flight Center and the Operational Land Imager (OLI) provided by Ball Aerospace and Technology Corporation (BATC) will form the payload for the Landsat Data Continuity Mission (LDCM). This paper will describe the design, capabilities and status of both the OLI and the TIRS instrument.

\begin{abstract}
The Landsat Data Continuity Mission (LDCM), a joint NASA and United States Geological Survey (USGS) mission, is scheduled for launch in December, 2012. The LDCM instrument payload will consist of the Operational Land Imager (OLI), provided by Ball Aerospace and Technology Corporation (BATC) under contract to NASA and the Thermal Infrared Sensor (TIRS), provided by NASA's Goddard Space Flight Center (GSFC). This paper will describe the design, capabilities and status of the OLI and TIRS instruments.

The OLI will provide 8 channel multispectral images at a spatial resolution of 30 meters and panchromatic images at 15 meter spatial resolution. The TIRS is a 100 meter spatial resolution push-broom imager whose two spectral channels, centered at 10.8 and 12 microns, split the ETM+ thermal bands. The two channels allow the use of the "split-window" technique to aid in atmospheric correction. The TIRS focal plane consists of three Quantum Well Infrared Photodetector (QWIP) arrays to span the $185 \mathrm{~km}$ swath width.

The OLI and TIRS instruments will be operated independently but in concert with each other. Data from both instruments will be merged into a single data stream at the (USGS)/Earth Resources Observation and Science (EROS) facility. The ground system, being developed by USGS, includes an Image Assessment System (IAS), similar to Landsat-7's, to operationally monitor, characterize and update the calibrations of the two sensors.
\end{abstract}

